\title{
Relief: Pictorial and otherwise
}

\author{
Jan J.Koenderink and Andrea J.van Doorn \\ Utrecht Biophysics Research Institute (UBI)
}

July 14, 1994

Surfaces play an important role in visual perception. They are perceived as "(perceptual) reliefs", that are surfaces in ${ }^{2+1} \mathrm{D}$ perceptual space, that is the product space of the ${ }^{2} \mathrm{D}$ visual field and the ${ }^{1} \mathrm{D}$ "depth dimension". It is in many respects irrelevant whether the observer views a true ${ }^{3} \mathrm{D}$ scene or a flat $\left({ }^{2} \mathrm{D}\right)$ picture of a scene. In both cases the percepts are reliefs in ${ }^{2+1} \mathrm{D}$ perceptual space. In the latter case one speaks of "pictorial relief". We discuss how perceptual reliefs can be measured and which aspects of these reliefs are especially robust against day to day intraobserver variations, changes of viewing conditions, and interobserver differences. It turns out that only aspects of the partial depth order (based on depth precedence in infinitesimal regions) are stable. Thus features of the relief are invariants of general "relief preserving transformations" that may actually scramble depth values at different locations. This is evident from the fact that human observers can only judge depth precedence with some degree of certainty for points that are on a single slope. We discuss the formal structure of these relief invariants. Important ones are the Morse critical points and the ridges and courses of the relief.

\section{The notion of "pictorial relief"}

The concept of "relief" originally derives from the arts of sculpting and painting. Sculpture designed for a particular vantage point (the generic situation for classical sculpture[22], e.g., pieces designed to be placed against a wall) need not be worked "in the round" in order to look natural, it can be squashed in depth to various degrees, even to almost flat work, and still appear as true threedimensional structure. Paintings are by nature totally "flat", nevertheless vivid relief may appear due to shading, etc. For instance, the work by the dutch painter Jacob de Wit[18] can hardly be distinguished from sculpted relief from its intended viewing position. (He made a successful living on this type of "illusion".) The concept of relief has been important in the theory of sculpture from renaissance times (vide Vasari's discusssion[21] of "Michelangelo's method"). Its general signifi- 
cance for the visual arts and indeed vision in general was recognized by the sculptor Adolf Hildebrand[7]. Hildebrand's book "Das Problem der Form in der bildenden Kunst" from 1893 has become extremely influential.

In Hildebrand's view there is indeed little difference between sculpted relief and "pictorial relief", that is the threedimensionality obtained from the viewing of pictures. In all these cases the observer is aware of a "depth flow", described by Hildebrand in terms of ridges (divides) and courses flowing around islands to the eventual depth of the background. The features he mentions ("ridges", "courses", "islands") appear as invariants of the depth flow that define the spatial shape relative to the observer.

It is easy enough to flesh out Hildebrand's intuitive notions with straight mathematics. However, in order to arrive at his invariant features one needs an idea of the types of transformations that conserve the relief. (Of course we have something of a chicken and egg problem here: In order to define the invariants we need the transformations, in order to know the transformations we need the invariants. Here we use both intuive notions as well as evidence from psychophysics to arrive at reasonable descriptions.) In computer vision one may think of an uncalibrated stereo rig that allows the construction of relief up to collinearities[5]. In this paper we are interested in the transformations that conserve pictorial relief for human observers. We use psychophysical methods to obtain an idea of these. Our resulting treatment is general though and applies equally well to various aspects of machine vision.

\section{Psychophysics}

In science we typically look for correlations between various physical entities. In the psychophysics of pictorial relief we have a physical entity (the ${ }^{2} \mathrm{D}$ picture) and a mental entity: The ${ }^{2+1} \mathrm{D}$ "percept" of an observer. In order to make science possible we have to find a physical entity that corresponds to the percept. Various methods are available, for instance, one may ask the observer to produce $\mathrm{a}^{3} \mathrm{D}$ replica of the percept (by kneading a lump of clay say). Other possibilities include the recording of answers to questions put to the observer that require reference to the percept $(e . g$., "is this angle-an angle is pointed out in the picture-acute or obtuse in $\left({ }^{3} \mathrm{D}\right.$ !) pictorial space?").

A very general method is the following: One places a test object ("gauge figure") in the scene (or a picture of a gauge figure in a picture of the scene, it makes-at least conceptually-no difference) and asks whether the test figure fits the scene in some a priori agreed way. This is typical for many physical measurements. For instance, in measuring length one places a yardstick next to the object and judges whether certain locations on the object coincide with certain marks on the yardstick. 


\subsection{Methods of probing pictorial relief}

One easily thinks of dozens of methods to probe various aspects of pictorial relief. We will discuss two examples here. In one example we place a gauge figure in the scene and ask the subject to adjust it to a perfect fit[8]. In the other method we indicate two points and ask for a relation between the pictorial relief at those points[9].

\subsubsection{Attitude probing}

Local surface attitude is the orientation of the local tangent plane relative to the vantage point. Convenient parameters are the slant, that is the angle the tangent plane subtends with the line of sight, and the tilt, that is the angle of the direction of steepest ascent along the tangent plane relative to a fiducial direction e.g., the vertical), measured in a plane orthogonal to the line of sight. Figure 1 illustrates this: The eye (e) views a surface element $(\mathcal{S})$. The slant $(\sigma)$ is the angle subtended by the surface normal (n) and the line of sight. The tilt $(\tau)$ is defined with respect to the fiducial orientation (r) in the frontoparallel plane. In figure 2 we depict a family of gauge figures parametrized in this way. If you superimpose a gauge figure (rendered as wireframe) over a picture surface (e.g., a photographic quality halftone rendering), most observers will readily tell you whether the gauge figure is in the pictorial surface or not.

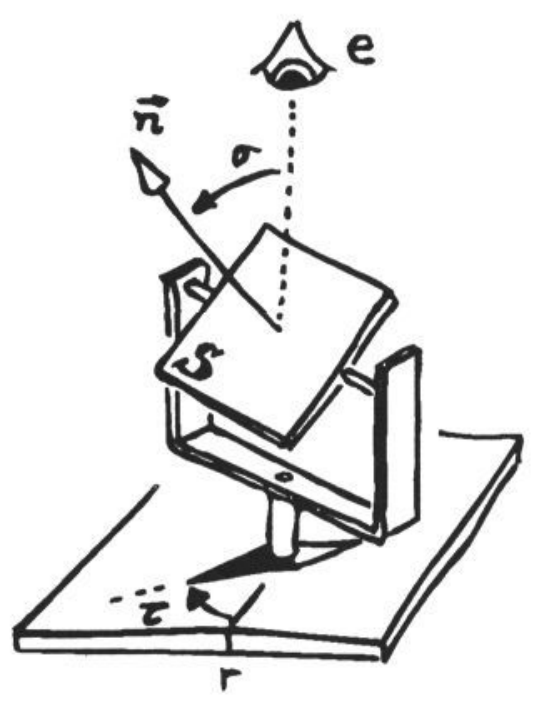

1. Slant and tilt.

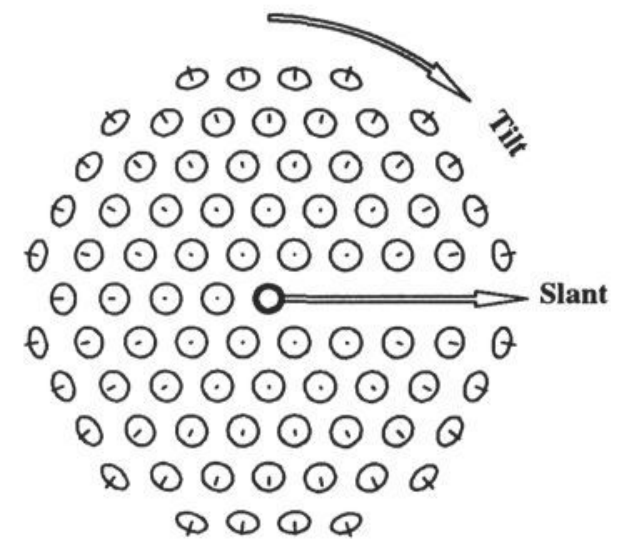

2. Gauge figures.

In this paper we report on a single stimulus, a monochrome photograph of a mannikin in en dos pose under fairly diffuse but somewhat directional, 
oblique illumination (Figure 3). The picture is rendered in 8 bit graytone on a CRT screen. (Figure 3 only approximates the original which is of photographic quality.) We triangulate the area in the picture that defines a certain pictorial surface, then superimpose gauge figures (rendered in red wireframe) on the picture centered at the vertices of this triangulation. (The subject is never directly aware of the triangulation, however, the triangulation determines where the probe will appear.) We visit the vertices in random order. For each vertex the subject adjusts the shape of the gauge figure so as to fit the pictorial surface. Subjects report that if the setting is satisfactory the gauge figure "sucks" to the surface and appears as a red circle painted upon it. Figure 4 shows the result of such a session (although all gauge figures are shown simultaneously in this figure, the subject saw only one at a time during the actual experiment!) for the picture shown in figure 3: We obtain a set of samples of the orientation of "the pictorial surface", or better, the measurements operationally define what we will subsequently call the "pictorial surface". We repeat this several times in order to obtain a measure of the standard error. Subjects can perform hundreds of such settings an hour. Afterwards we analyze the resulting discrete field of pictorial surface attitudes.

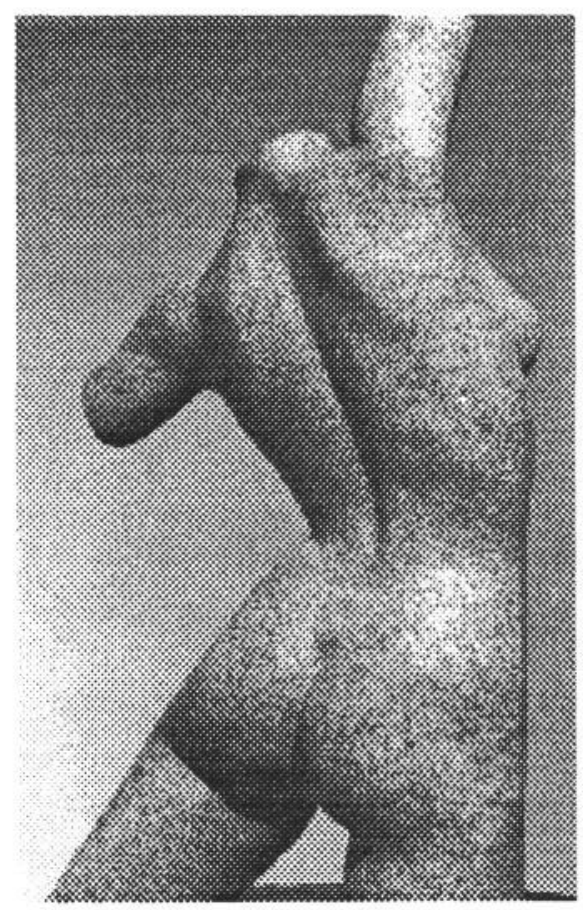

3. Stimulus picture.

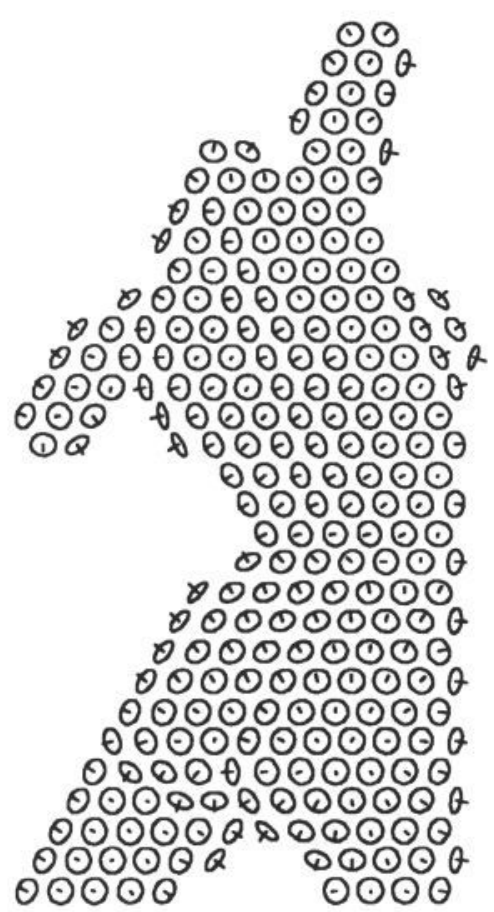

4. Attitude field. 
We find that the empirical field of attitudes is indeed consistent with a surface interpretation (a gradient field, vanishing curl). This is most remarkable in itself and indicates that a " $2 \frac{1}{2} \mathrm{D}$-sketch" model[15] does not apply. In a ${ }^{2 \frac{1}{2}} \mathrm{D}$-sketch one obtains a set of mutually independent local estimates. Chances are slim that such a discrete field will turn out to be fully consistent with any surface. Of course the statement that "the empirical field of attitudes is indeed consistent with a surface interpretation" needs to be backed up with statistical arguments. We have reported on this elsewhere[8]. Just for reference, the total depth variation over a face of the triangulation ranges from zero to up to 60 pixels (in this method we measure depth in the same units as distance in the frontoparallel plane!), whereas the violations over the boundary of a face are typically of subpixel dimensions.

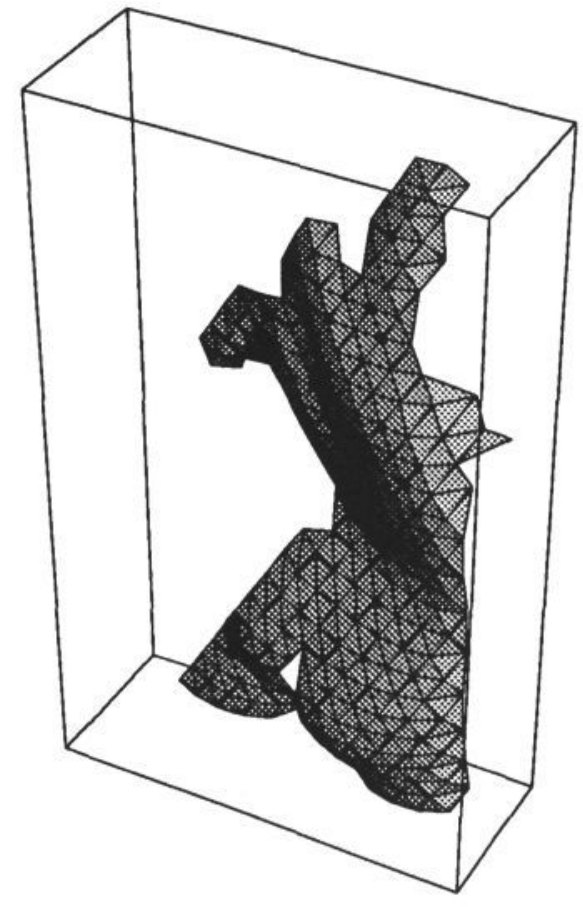

5. Pictorial relief.

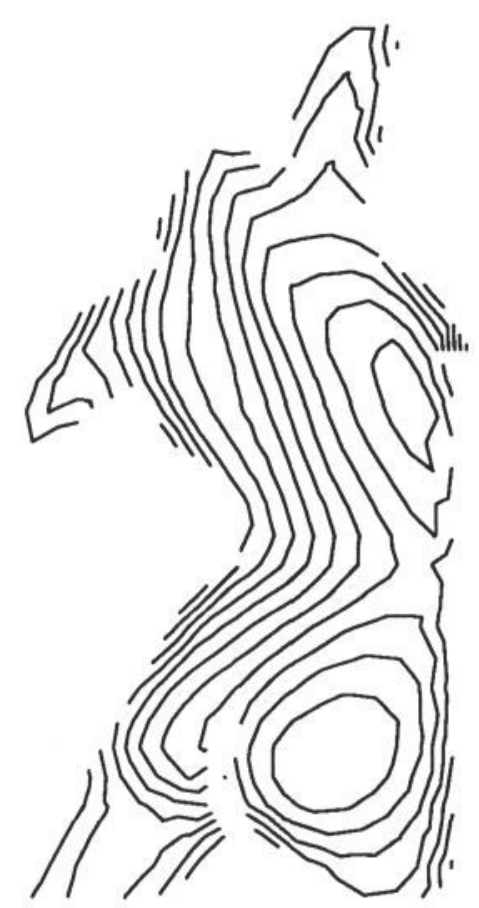

6. Depth isocontours.

As a consequence of this surface consistency we may proceed to obtain the $\left({ }^{3} \mathrm{D}\right.$ !) pictorial relief by integration of the field of attitudes. Figure 5 shows a generic view of the pictorial surface. Notice that we have transformed the ${ }^{2} \mathrm{D}$ picture into a surface immersed in ${ }^{3} \mathrm{D}$ by this psychophysical method. In figure 6 we show a congruence of isodepth curves that perhaps defines the result somewhat more precisely. From such data we may compute profile renderings and thus confront the subjects with their own depth 
image translated into frontoparallel distances. Subjects are typically satisfied with the result, whatever value one may attach to this observation.

\subsubsection{Effects of viewing conditions and variation over subjects} Different subjects produce different surfaces. Moreover, even a single observer produces different surfaces when the viewing conditions are varied (e.g., monocular versus binocular, or frontal versus oblique viewing). For both inter- and intra-observer variations we find that typically the results differ by a depth scaling which is often quite large (up to a factor of five). The depth scale appears to be a rather "elastic" one[11]. Moreover, when we analyse the day to day variations in settings it turns out that most of the variance is in the depth dimension: Subjects are dead sure about the tilt, but sloppy in the slant domain. This is equally true for intersubject comparisons: Subjects tend to agree on the tilt but differ on the slant.

In the interobserver comparisons we sometimes find ideosyncratic deviations that are apparently not of a stochastic character. For instance, one subject may produce an additional twist of the lumbar with respect to the pelvic region as compared to another one.

\subsubsection{Depth precedence probing}

We superimpose two distinguishable (e.g., through their hue) dots on the picture and require the subject to answer which one is closer (forcing unambiguous answers). Repeated trials allow the determination of the probability of the first dot being judged closer than the second dot for a great number of ordered dot pairs. (In practice several professional psychophysical tricks have to be played, for instance one has to balance presentations to allow for the fact that some hues have a tendency to look closer than other ones. We skip many of such conceptually trivial-though practically crucial-psychophysical details in this paper!)

When we perform this task on the edges of the triangulation (of course again visited in random order) we obtain a probability of the depth precedence conforming to the orientation of the edge for each edge. Assuming a fixed depth difference limen we may convert these probabilities to depth differences between the endpoints of the edge (at least if the probabilities are not identically zero or one). Again, we may test for surface consistency (for $(\mathcal{A} \rightarrow \mathcal{B}) \wedge(\mathcal{B} \rightarrow \mathcal{C})$ should imply $\mathcal{A} \rightarrow \mathcal{C}$, where the arrow indicates depth precedence). We find perfect consistency within the experimental tolerances. This means, of course, that the judgments for different point pairs are by no means independent: They are constrained by something not completely local, the pictorial relief.

We end up with many (perhaps a 1000) mutually consistent depth difference inequalities and equalities and may proceed to solve this set for the pictorial relief[9]. (In practice we use the method of iterative projection on 
convex sets to solve this problem. In theory at least linear programming would also work.) Figure 7 shows such a pictorial surface reconstructed from depth order judgments. In figure 8 we show the corresponding congruence of isodepth curves.

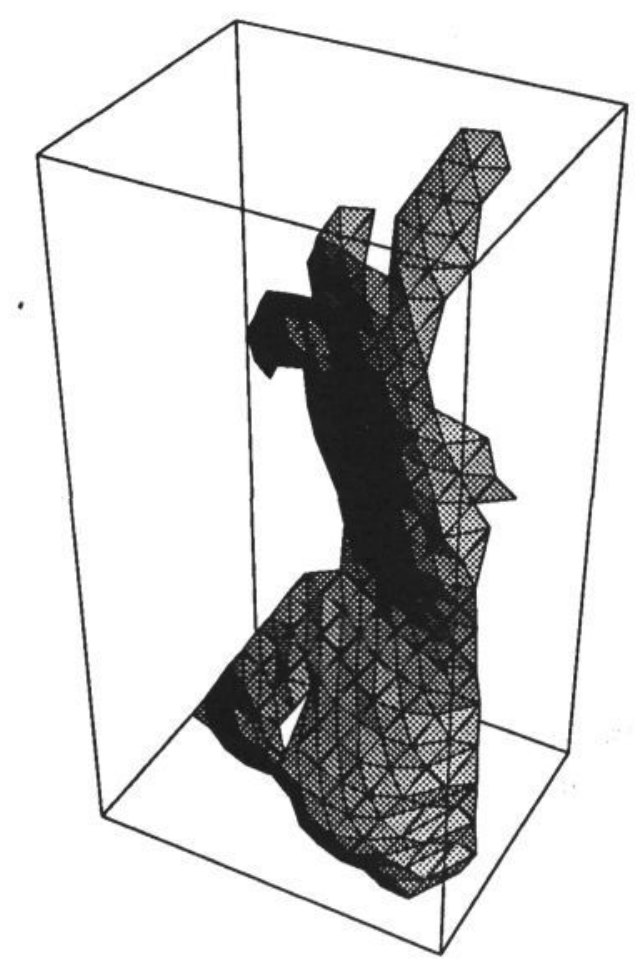

7. Pictorial relief.

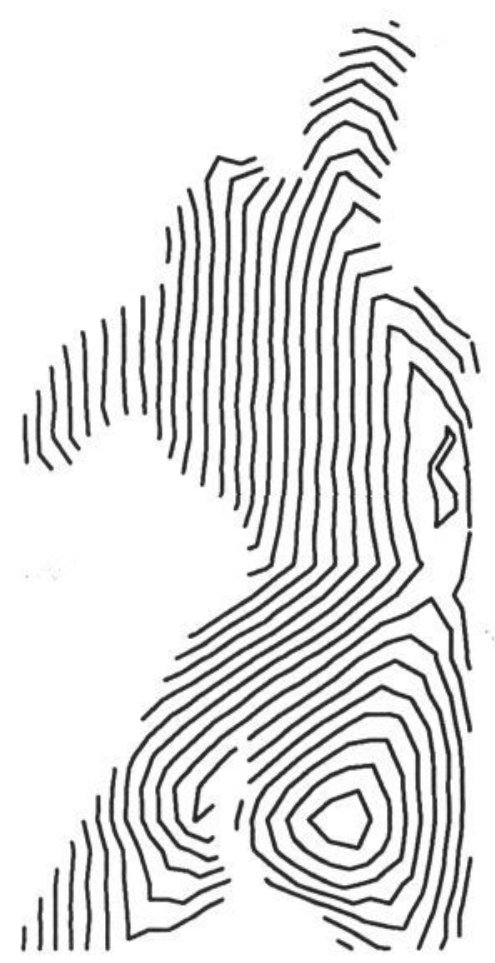

8. Depth isocontours.

\subsubsection{Depth precedence judgments for large separations}

Although observers readily judge depth precedence if the two dots are not very widely separated in the picture, they often tend to hesitate or merely guess if the pair is widely separated in the frontoparallel plane. We have measured probabilities of judging every vertex (not just the nearest neighbors) of the triangulation as closer than a (limited) number of fiducial vertices. (The judgments were done in interleaved fashion in randomized order.) Figures 9, 12 and 15 show the result for such an experiment for three particular fiducial vertices (these vertices are most easily identified in figures 10 and 11, 13 and 14, 16 and 17). Notice that it would be out of the question to measure all combinations: The subject would have to perform millions of judgments, consuming thousands of hours. 

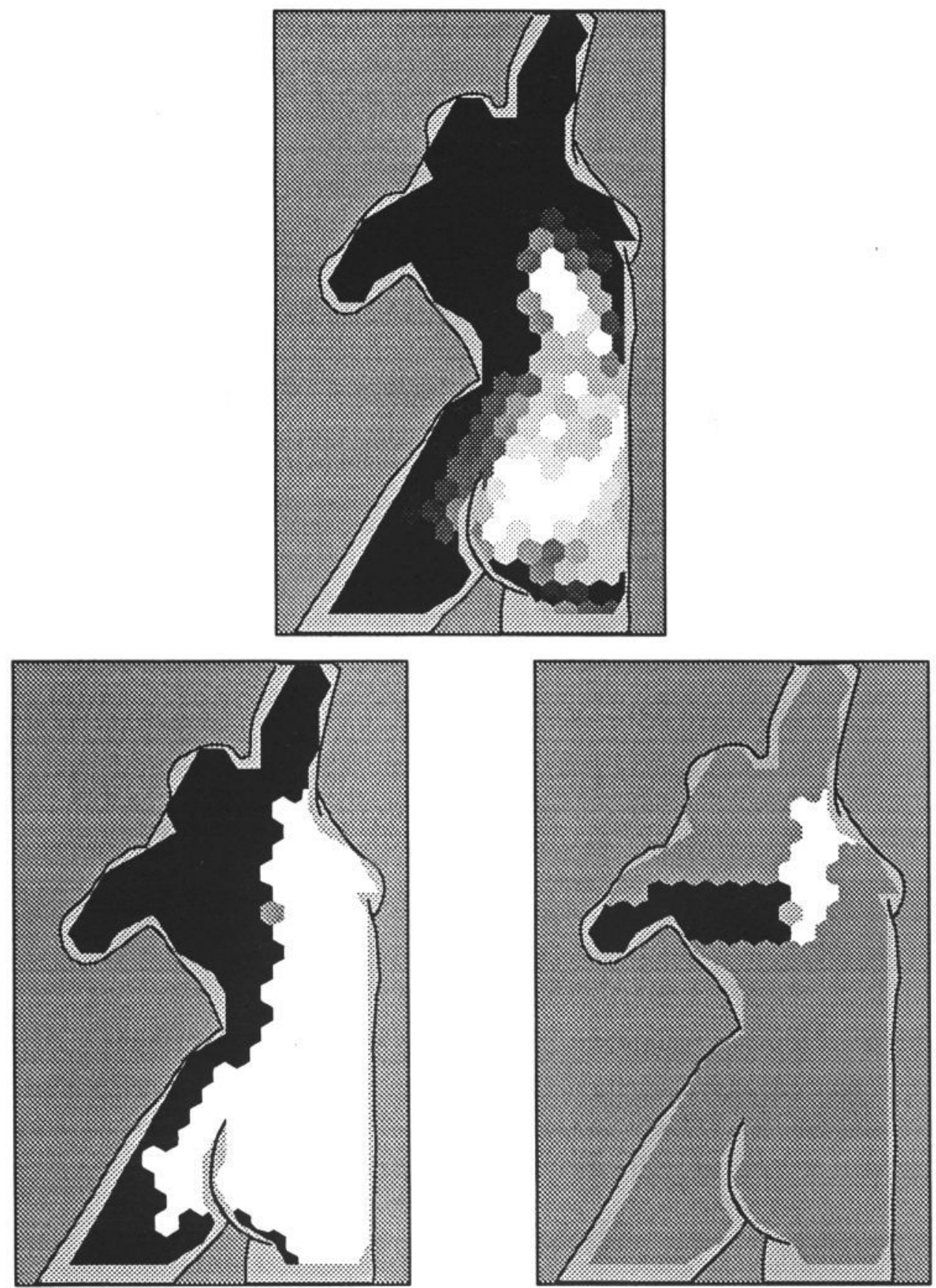

Figures 9-11: Fiducial vertex \#101. Upper: 9. Probabilities (graylevel indicates probability of being in front. White $=1$, Black $=0$ ), Lower left: 10. Thresholded pictorial relief (White=hither, Black=yonder), Lower right: 11. Prediction from partial depth order (White=hither, Black=yonder, Gray $=$ don't know). 

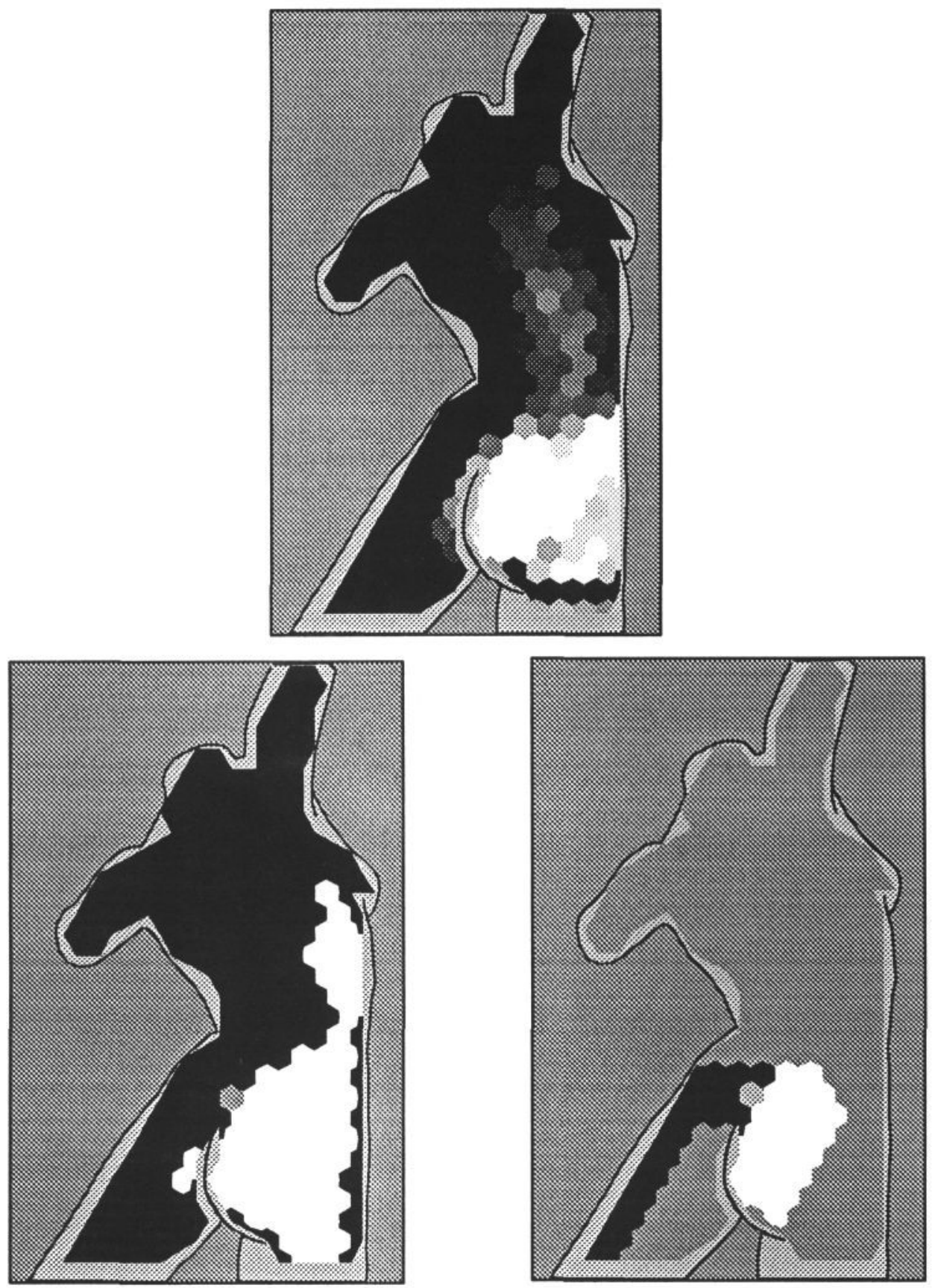

Figures 12-14: Fiducial vertex \#156. Upper: 12. Probabilities (graylevel indicates probability of being in front. White $=1$, Black $=0$ ), Lower left: 13. Thresholded pictorial relief (White=hither, Black=yonder), Lower right: 14. Prediction from partial depth order $($ White $=$ hither, Black=yonder, Gray $=$ don't know). 

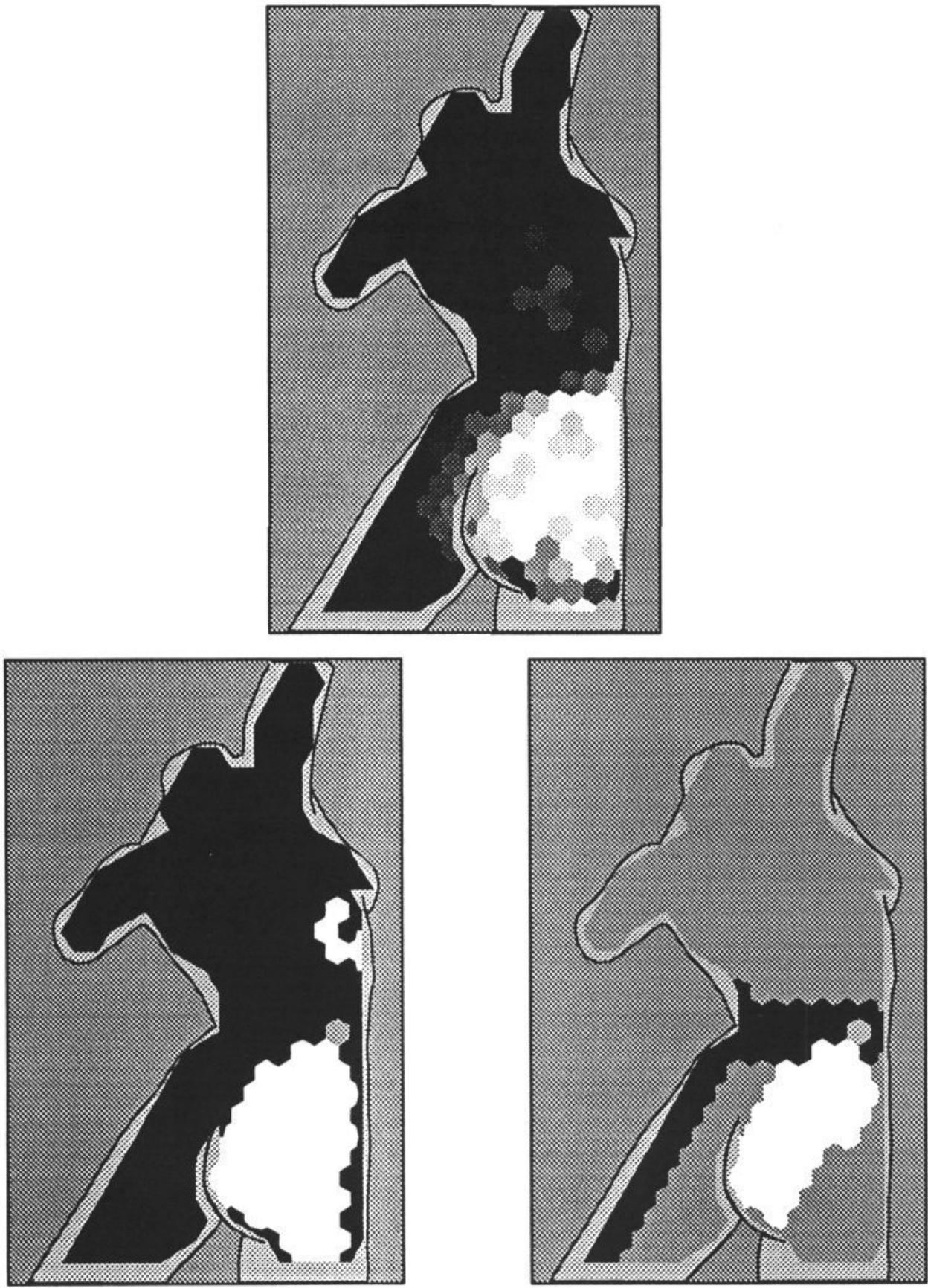

Figures 15-17: Fiducial vertex \#211. Upper: 15. Probabilities (graylevel indicates probability of being in front. White $=1$, Black $=0$ ), Lower left: 16 . Thresholded pictorial relief (White=hither, Black=yonder), Lower right: 17. Prediction from partial depth order (White=hither, Black=yonder, Gray $=$ don't know). 
These results may be compared with the pictorial relief obtained by other methods thresholded with respect to the depths of the fiducial vertices. Figures 10,13 and 16 (fiducial vertices \#101, \#156 and \#211 respectively) show such decompositions in "hither" and "yonder" areas of the pictorial surface. One would perhaps expect subjects to respond on this basis, after all the pictorial relief has been obtained from subject judgments in the first place, thus one expects subjects should be in the possession of this information. However, we find that subjects fail miserably on this task. In many cases they cannot judge depth precedence of two points reliably, despite the fact that the depths are well separated in the pictorial relief. Apparently we have detected a rather serious limitation of the human mind here.

It would appear that human observers can only judge depth precedence for points that are on a single slope, not if the points are separated by (we use the terms in an informal sense at this place) a ridge or course. Indeed, if we don't threshold the relief surface (which is a global method in the sense that one assumes that the depths at arbitrarily separations can be judged) but follow the empirically established depth precedence order for the edges (this is very much like keeping only track of the direction of the depth gradient) the predictions conform somewhat better to our findings. (Notice that this method is purely local in the sense that only closely spaced points need be compared at any time.) Figures 11, 14 and 17 show this prediction for the examples presented here. The picture is parsed in a "hither", a "yonder" and a "somewhere" (or "don't know") region. Notice how-at least by "eye-measure" - this prediction conforms more closely to the actual data (figures 9,12 and 15) than the thresholded pictorial reliefs (figures 10,13 and 16) do. It is not so easy to back this up with numbers though. We may for instance correlate the predictions with the empirical probabilities. For the predictions via the thresholded relief the correlation extends over all vertices (251), for the predictions via the partial depth order the don't knows are not included in the correlation. Correlations are: \#101, correlation with thresholded relief 0.73 , with partial order 0.53 (based on 36 samples); \#156, correlation with thresholded relief 0.69 , with partial order 0.92 (based on 50 samples); \#211, correlation with thresholded relief 0.77 , with partial order 0.88 (based on 72 samples). The predictions from the partial depth order are significantly better except for the fiducial vertex \#101, for which both correlations are fairly low. However, one really needs an in depth analysis of the residuals to make sense of these numbers and a perusal of the patterns apparent in figures 9-17 will do as well.

Clearly the actual result is somewhat "in between" these two extreme predictions, tending perhaps more closely to the prediction from the local depth order alone. Apparently observers can do linear depth order judgments to some extent, though they are doubtless bad at it. On the other hand the partial depth order is readily available to the observers. 
The failure of the human observer to make effective use of the slope magnitude and the observer's sole reliance on local depth order have been predicted more than a decade ago[11]. This was first empirically demonstrated by Todd and Reichl[20], though these authors lacked our present powerful methods to probe pictorial relief.

\section{Invariants of relief preserving transformations}

In this section we change gears and discuss invariants of relief preserving transformations in the formal sense. We use the psychophysical results (v.s.) to guide our selection of interesting transformations.

Classical "relief preserving transformations" are collineations that move the plane at $\infty$ to a frontoparallel plane at some arbitrary distance. Such transformations have been used in the design of stage decors where one meets the problem of accommodating all the space in front of an observer in some finite depth slice. The method is at least two centuries old and is mentioned in the $19^{\text {th }} \mathrm{c}$. vision literature[6].

Special cases include depth scalings. Apparently depth scalings describe the differences between many of our psychophysical results quite well.

More general transformations that might be said to "preserve relief" are general monotonic transformations of the depth scale. Such transformations are quite common in the image intensity domain: If you display a picture on different monitors then the photometric radiances at corresponding points are-almost certainly - related by some nonlinear monotonic transformation. So called "gamma corrections" are just power functions of this type.

One may consider even more general transformations for which it might be said that-in a certain sense-that they "conserve relief". Suppose I hand you a depth gradient field, but suppress slope information (i.e., specify only the field lines). Then the surfaces that are described by this information are in general not related by any monotonic transformation of the depth scale. In fact, depths at different points (in the frontoparallel plane) may be scrambled.

If the gradient direction field is $\mathbf{e}(\mathbf{x})($ with $\mathbf{e . e}=1)$, then a field $\mathbf{g}(\mathbf{x})=$ $s(\mathbf{x}) \mathbf{e}(\mathbf{x})$ (for some slope function $s$ ) is a gradient field if the curl vanishes, i.e., if $\nabla \log s \wedge \mathbf{e}+\nabla \wedge \mathbf{e}=0$. Thus $\log s$ has to satisfy a linear partial differential equation determined by the gradient direction field. Though this severely constrains the possibilities, there is still quite a bit of leeway left.

If the relief is subject to such general relief preserving transformations we cannot find a linear order for the points (on the basis of depth precedence), instead one obtains merely a partial order. The points can be divided into "natural districts" (hills and dales[3,16]) in such a way that all 
points of a dale are above its immit, whereas all points of a hill are below its summit. Figure 18 shows an example of a slope defined by a single summit (disk) and a single immit (open circle). (In this case the boundary of the slope contains two saddle points (circles with crosses), some slopes contain only a single saddle though!) All the slopelines run between summit and immit and are homotopic; this is the largest possible region of this nature and thus represents the natural definition of the intuitive notion of "a slope". The union of all slopes on a summit form a "hill", of all slopes on an immit a "dale": Thus the landscape naturally divides into hills or in dales. These are Maxwell's "natural districts" and are the formal equivalents of Hildebrand's "islands".

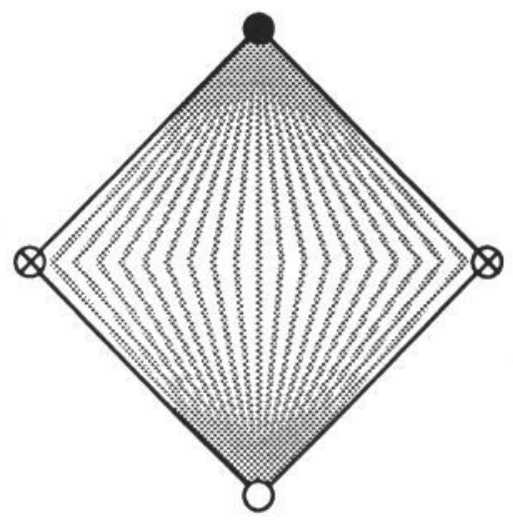

18. Schematic slope.

\subsection{Isocontours, slope lines and Morse critical points}

General relief preserving transformations are evidently defined by the fact that they conserve the curvilinear congruence of isocontours (loci of equal depth[14]), or-equivalently-the slopelines, that are the integral curves of the depth gradient field. Notice that though the natural order of the isocontours is conserved, the actual labels of the isocontours are lost.

Clearly the immits, summits and saddles are also conserved, since they are just singular isocontours. These topological invariants of general relief preserving transformations are the so called Morse critical points[17]: The extrema (near- and far-points) as well as the saddlepoints of the depth.

\subsection{Ridges and courses}

Apart from the Morse critical points there also exist invariant curves, the so called ridges and courses of the relief. These are defined as certain singular solutions of the slope line equation. In short, if $z(x, y)$ denotes depth as a function of the Cartesian coordinates $(x, y)$, then the slopeline equation 
is $q(x, y) d x-p(x, y) d y=0$, with $p(x, y)=\frac{\partial z}{\partial x}$ and $q(x, y)=\frac{\partial z}{\partial y}$. Let its general solution be $w(x, y)$. Then we introduce a function $\vartheta(x, y)$ such that $\vartheta(x, y) d w=q(x, y) d x-p(x, y) d y$, thus $\vartheta$ is an integrating divisor of the slope line equation. (Notice that the one-form[2] $d w$ cannot possibly be exact.) The curves $\vartheta(x, y)=0$ with the additional condition $d w \wedge d \vartheta=$ $\left(\frac{\partial w}{\partial x} \frac{\partial \vartheta}{\partial y}-\frac{\partial w}{\partial y} \frac{\partial \vartheta}{\partial x}\right) d x \wedge d y \neq 0$ are singular solutions of the slopeline equation that possess the intuitive properties of "ridges" and "courses". For instance, the slopelines approach a course from both sides and the direction of the slopelines approaches that of the course arbitrarily close. One may impose additional constraints, e.g., that the slopelines should approach the course asymptotically, otherwise one might obtain an extended marshland instead of a narrow rivulet.

The definition (essentially $\vartheta=0$ ) of ridges and courses is based upon the same physical principle (local extremal slope along an isocontour) as the classical definition[4]. However, one usually derives a nongeneric (and generally false) conclusion from this (thus essentially following de SaintVenant[4]'s reasoning). That this method is not correct is evident from the fact that the solutions of de Saint-Venant's differential equation are--in the general case-curves that are transverse to the slope lines. This violates a simple principle (due to Boussinesq[1]) that water always runs downhill: Thus the direction of the ridges and courses should everywhere coincide with that of the slope lines. However, this doesn't constrain the ridges and courses to be slopelines, Boussinesq's condition is satisfied by the singular solutions too.

The present definition coincides with the classical one (solutions of de Saint-Venant's differential equation) for the special case of straight courses and ridges. Although the correct solution was already published (by Rothe[19]) in 1915, the erroneous solution still runs at large. (See discussion in Koenderink et $a l[12,13]$.) The various so called "ridge finders" from the literature of computer vision and image processing either are tweaked to find entities quite unlike the topographical ridges or simply compute the wrong thing (that is: the de Saint-Venant's solution, though usually without reference). This is illustrated in figures 19 and 20 . Figure 19 shows the topography (isocontours) of the landscape $z(x, y)=$ $\arctan \frac{y}{x}+\frac{1}{2}\left(\sqrt{x^{2}+y^{2}}-1\right)^{2}$. This "landscape" is actually a helicoïdal gutter, a bit like an infinite parking garage. The thick circle (unit radius) is the course, the thin circle (radius $1.38028 \ldots$...) is the solution of de SaintVenant's equation. Figure 20 shows the depth flow. Notice that the flow crosses the "official solution" (de Saint-Venant's equation) transversely, thus violating Boussinesq's condition[1] (the water doesn't run downhill but rather takes an oblique course). The flow asymptotically approaches the course and eventually everything is bound to join this streambed.

Notice that the ridges and courses are in many respects reminescent of one-dimensional analogues of the (zero-dimensional) Morse critical points. 
The Morse critical points define the sources and sinks and the ridges and courses the separatrices of the slope field or "creep".

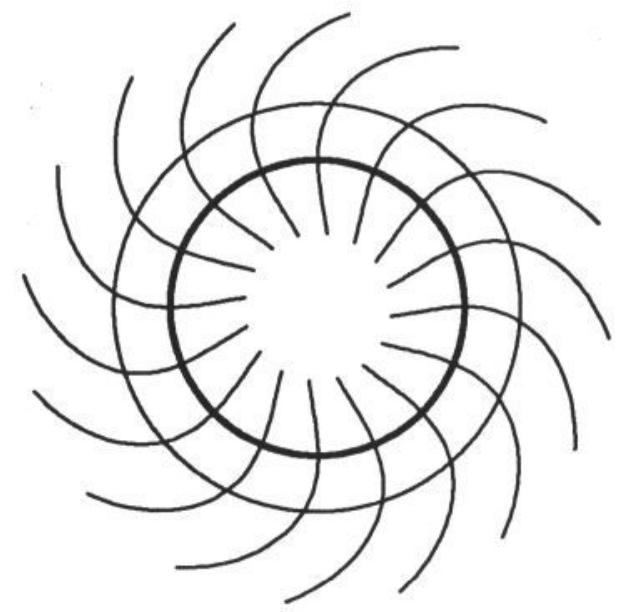

19. Topography.

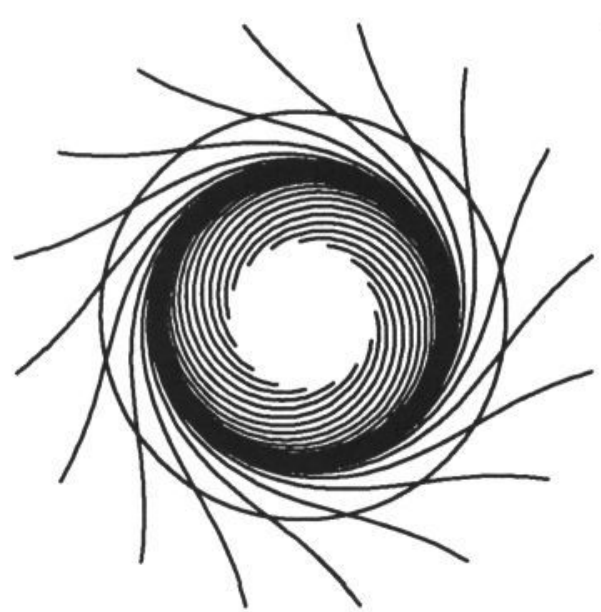

20. Depth flow.

In figures 21 and 22 (next page) we show the isodepth curves, Morse critical points (circles with center dots: far points, circles with center circles: near points, circles with crosses: saddles), ridges (black linesegments) and courses (gray line segments) for the pictorial reliefs obtained from attitude gauge figure adjustments and depth precedence judgments. They are seen to be very similar, though subtle differences exist. Major near points define the right shoulder blade and the buttocks, far points occur mainly on the boundary of the triangulation, alternating with saddles which are near points of that boundary. An internal saddle is seen in the middle of the figure. The main ridge defines the vertical body axis, minor ridges define the shoulder girdle and the arms and legs. The major course runs on the back and separates the thorax from the pelvic region. These relief structures formally describe the "depth flow" as considered intuitively by Hildebrand[7].

\section{Discussion}

We have presented psychophysical evidence for the fact that human observers can address globally consistent range maps and/or attitude fields, thus clearly refuting the utility of the hypothesis of the classical " $2 \frac{1}{2} \mathrm{D}$ sketch" for the interpretation of our psychophysical results. However, we have also shown that human observers are not able to use these datastructures in a fully general way. Apparently they can only establish depth precedence to any reasonable extent if the locations to be compared are 
members of a single hill or dale, more likely even members of the points common to a certain hill and a certain dale, or in other words "a single slope".

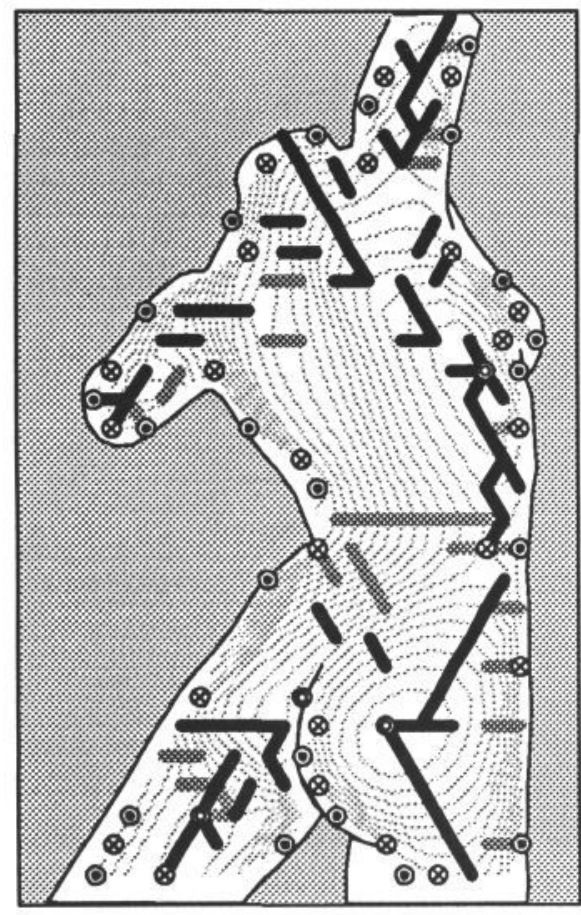

21. Refief from attitudes.

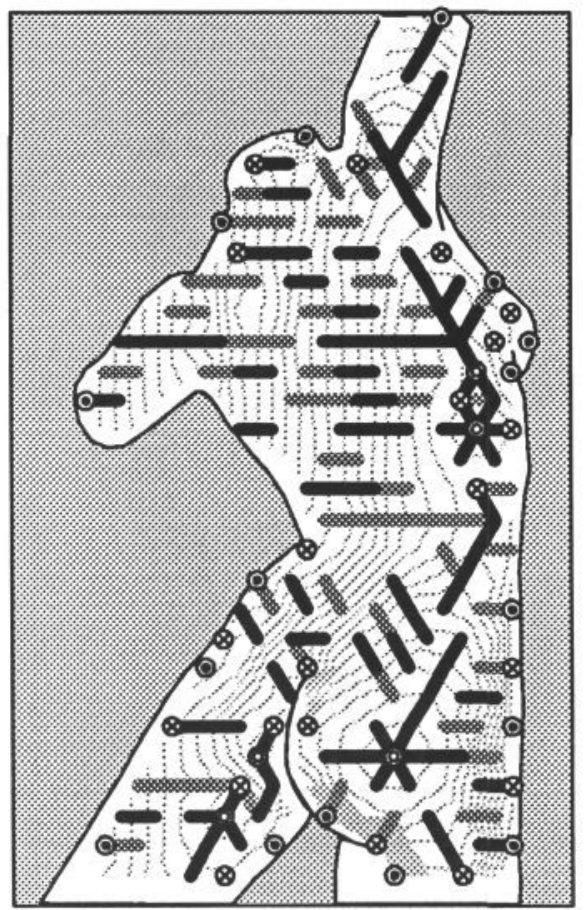

22. Relief from depth order.

We have briefly indicated the mathematical framework needed to deal with these types of datastructures. The "relief" available to human observers is only fully specified up to arbitrarily "general relief preserving transformations", that are transformations than merely conserve the partial order defined by the hills and dales. Important topological invariants of these transformations are the Morse critical points and the courses and ridges. These are a priori important "features" of pictorial relief.

Of course there also exist metrical invariants, such as the curvature of the isocontours and slopelines. In the literature on image processing the loci of extremal curvature along the isocontours have been implied as natural courses and ridges. However, it is easily demonstated that such loci are not necessarily separatrices of the creep at all and that they in general violate Boussinesq's condition[1]. The relevance of these invariants for human perception remains as yet unsettled.

ACKNOWLEDgements This work was done in a Human Frontier Science Program project sponsored by the Japanese government. 


\section{References}

[1] Boussinesq, J., "Sur une propriété remarquable des points où les lignes de plus grand pente d'une surface on leur plans osculateurs verticaux, et sur la différence qui existe généralement, à la surface de la terre, entre les lignes de faîte et de thalweg et celles le long desquelles la pente du sol est un minimum", C.R.Paris 73, p.1368, 1871.

[2] Cartan, H., Differential forms, Hermann, Paris, 1983.

[3] Cayley, A., "On contour and slope lines", The London, Edinburgh and Dublin Philisophical Magazine and J. of Science, Vol. 18, No. 120, pp. 264-268, 1870.

[4] De Saint-Venant, "Surfaces à plus grande pente constituées sur des lignes courbes", Bulletin de la soc.philomath. de Paris, March $6^{\text {th }}$, 1852 .

[5] Faugeras, O.D., What can be seen in three dimensions with an uncalibrated stereo rig, In: Giulio Sandini, ed., Proc.2nd Eur.Conf. Computer Vision, Springer-Verlag, 1992.

[6] Helmholtz, H.von, Handbuch der physiologischen Optik, 2nd. ed., Verlag von Leopold Voss, Hamburg and Leipzig, 1896.

[7] Hildebrand, A., Das Problem der Form in der bildenden Kunst, Strassburg, 1893.

[8] Koenderink J.J., A.J.van Doorn and A.M.L.Kappers, "Surface perception in pictures", Perception and Psychophysics 52, pp. 487-496, 1992.

[9] Koenderink J.J., A.J.van Doorn and A.M.L.Kappers, "Pictorial surface attitude and local depth differences compared", UBI-TR, 1994.

[10] Koenderink J.J., A.J.van Doorn and A.M.L.Kappers, "On so called paradoxical monocular stereoscopy", Perception, in press, 1994.

[11] Koenderink J.J. and A.J.van Doorn, "The Structure of twodimensional scalar fields", Biol. Cybernetics 33, pp. 151-158, 1979.

[12] Koenderink J.J. and A.J. van Doorn, "Two-plus-one-dimensional differential geometry", Pattern Recognition Letters 15, pp.439-443, 1994 .

[13] Koenderink J.J., and A.J. van Doorn, Local Features of smooth shapes: Ridges and courses, in: Geometric methods in computer vision II, ed. B.C.Vemuri, SPIE Vol. 2031, pp.212-223, 1993. 
[14] Liebmann, H., Geometrische Theorie der Differentialgleichungen: Die topographischen Kurven, in: Encyklopädie der Mathematischen Wissenschaften mit Einschluß ihrer Anwendungen, Vol. III, $3^{\text {rd }}$ part, W.Fr.Meyer and H.Mohrmann (eds.), B.G.Teubner, Leipzig, 19021927.

[15] Marr, D., Vision, W.H. Freeman and company, San Francisco, CA, 1982.

[16] Maxwell, J.C., "On hills and dales", The London, Edinburgh and Dublin Philisophical Magazine and J. of Science, Vol. 40, No. 269, pp. 421-425, 1859.

[17] Morse, M. and S.S.Cairns, Critical point theory in global analysis and differential topology, Academic Press, New York and London, 1969.

[18] Rosenberg, J., S.Slive and E.H.ter Kuile, Dutch art and architecture 1600-1800, Penguin Books, Harmondsworth, Middlesex, 1966.

[19] Rothe, R., "Zum Problem des Talwegs", Sitz.ber. d. Berliner Math. Gesellschaft, 14, pp. 51-69, 1915.

[20] Todd, J.T. and F.D. Reichel, "Ordinal structure in the visual perception and cognition of smooth surfaces", Psychologocial Review 96, pp. 643-657, 1989.

[21] Vasari, G., The lives of the painters, sculptors and architects, Translated by A.B.Hinds, Everyman's Library, Dutton New York, Part III, Michelagnolo Buonarotti, pp.108-192, 1963 (orig. 16th c.).

[22] Wittkower, R., Sculpture, processes and principles, Harper and Row, New York, 1977.

Author's address:

Prof.Jan J. Koenderink, D.Sc.

Utrecht Biophysics Research Institute (UBI),

Buys Ballot Laboratory,

P.O. Box 80000

NL-3508 TA Utrecht

the Netherlands 